\title{
Retinal nerve fibre layer thickness in a normal black South African population
}

\author{
Sarah Ismail ${ }^{1} \cdot$ Naseer Ally $\mathbb{1 0}^{1} \cdot$ Hassan Dawood Alli ${ }^{1}$ \\ Received: 23 June 2019 / Accepted: 24 October 2019 / Published online: 13 November 2019 \\ (c) The Author(s), under exclusive licence to The Royal College of Ophthalmologists 2019
}

\begin{abstract}
Background The measurement of retinal nerve fibre layer (RNFL) thickness on spectral domain OCT (SD-OCT) are compared with built-in age - and gender-matched European normative databases and this difference is used to assist with glaucoma diagnosis. However, there are differences in RNFL thickness between population groups. Therefore, using the built in European normative database as a comparison across all population groups could lead to erroneous results, due to the basic assumption that the normative values for non-European populations are the same as their European counterparts.

Methods Cross-sectional study of RNFL thickness in normal black South African patients.

Results One hundred and thirty-two eyes of 132 patients were enroled in this study. The mean (SD) age of patients in this study was $41.3(12.5)$ years. Males comprised $40.9 \%(n=54 ; p=0.0367)$. All RNFL sectors except the temporal sector were significantly thicker than the reference database. The RNFL sectors measured as follows: global $(108.7 \mu \mathrm{m}, p<0.001)$, superotemporal (152.4 $\mu \mathrm{m}, p<0.001)$, superonasal (132.6 $\mu \mathrm{m}, p<0.001)$, inferotemporal $(150.1 \mu \mathrm{m}, p<0.001)$, inferonasal (129.2 $\mu \mathrm{m}, p<0.001)$, nasal (77.7 $\mu \mathrm{m}, p<0.001)$, temporal $74.8 \mu \mathrm{m}, p=0.9534)$.

Conclusion The RNFL thickness of normal black South Africans is significantly thicker than that of the European database on the Spectralis SD-OCT. This needs to be taken into account when performing RNFL thickness measurements on black patients.
\end{abstract}

\section{Introduction}

Worldwide, glaucoma is known to be the leading cause of irreversible blindness with the number of people above 40 years of age living with the disease projected to increase from 76 million in 2020 to 111.8 million in 2040 [1]. In African patients, glaucoma is 4-5 times more prevalent than Europeans, occurs $~ 10$ years earlier and is more aggressive $[2,3]$. Multiple imaging modalities are used to detect early structural glaucomatous damage.

Technological advances in imaging quality and accuracy have resulted in older modalities (Heidelberg Retinal Tomogram and Time Domain Optical Coherence Tomography) becoming obsolete and newer modalities such as Spectral Domain Optical Coherence Tomography (SDOCT) and Optical Coherence Tomography Angiography

Naseer Ally

naseerally@gmail.com

1 Department of Neurosciences, Division of Ophthalmology, University of the Witwatersrand, Johannesburg, South Africa
(OCTA) being at the forefront in detecting pre-perimetric glaucoma. SD-OCT can be used in several ways to assist in glaucoma diagnosis; such as measurements of peripapillary retinal nerve fibre layer (RNFL) thickness, macular thickness and measurement of Bruch's-Membrane OpeningMinimum Rim Width (BMO-MRW) [4].

The measurement of RNFL thickness on SD-OCT are compared to built-in age - and gender - matched European normative databases and this difference is used to assist with glaucoma diagnosis [5]. However, there are differences in RNFL thickness between population groups [6-11]. Therefore, using the built in European normative database as a comparison across all population groups could lead to erroneous results, due to the basic assumption that the normative values for non-European populations are the same as their European counterparts. In addition, these databases are also machine-specific and not universally applicable between different types of SD-OCT machines (e.g. Spectralis $^{\mathrm{TM}}$, Cirrus $^{\mathrm{TM}}$, OptoVue ${ }^{\mathrm{TM}}$, Topcon $^{\mathrm{TM}}$ ) [12-15].

Studies using the Spectralis SD-OCT (Heidelberg Engineering, Germany) to ascertain the normal RNFL thickness compared to the built-in European normative database, have been conducted in numerous countries including Germany, 
India, Brazil, Nepal and in the French Alienor population $[6,8-10,16]$. These studies have shown a thicker RNFL in non-European populations [8-10]. In two Nigerian populations and South Africa, a thicker RNFL has been shown using the Stratus ${ }^{\mathrm{TM}}$ OCT and the Ivue- $100^{\mathrm{TM}}$ SD-OCT machines respectively $[11,17-20]$.

The aim of this study was to compare the normal retinal nerve fibre layer thickness in a healthy black population to the European normative database on the Spectralis SD-OCT (Heidelberg engineering). To the authors knowledge, this is the first study conducted in Africa using the Spectralis SDOCT (Heidelberg engineering).

\section{Materials and method}

We conducted a cross-sectional study at St John Eye Hospital - Chris Hani Baragwanath Academic Hospital in Soweto, Johannesburg, South Africa, from January 2017 to December 2018. The Human Research Ethics Committee of the University of the Witwatersrand, Johannesburg, gave ethical clearance for this study to be performed. The study adhered to the tenets of the Declaration of Helsinki. Staff, patients and their family members were invited to participate in the study. A convenient sample was taken from the outpatients' department at St John Eye Hospital. Each participant in the study gave written informed consent. Participants who met the following inclusion criteria were enroled in the study:

- Self-reported identity of black South African ethnicity,

- 18 years or older,

- Uncorrected Snellen visual acuity of $6 / 9$ or better in the study eye,

- Normal intraocular pressure (10-21 mmHg) with either Perkins or Goldmann applanation tonometry,

- Clinically normal optic disc,

- Absence of other fundus pathologies,

- Fovea to Disc (FoDi) alignment within $7^{\circ}$.

Exclusion criteria included patients with:

- Systemic diseases such as diabetes mellitus and neurological disease with optic disc abnormalities.

- Ocular conditions such as glaucoma, tilted discs, peripapillary atrophy, clinically significant vitreal, retinal or choroidal diseases; history of cataract or posterior segment surgery and laser procedures; prior or current history of eye trauma or uveitis; and grade three and four hypertensive changes were not included in the study.

- Axial length $>26.0 \mathrm{~mm}$.
The participants' demographic (age and gender) and medical history were documented. A Snellen visual acuity measurement at $6 \mathrm{~m}$; slit lamp examination; intra-ocular pressure measurement; and dilated fundoscopy (to assess cup:disc (C:D) ratio and exclude other pathologies) were performed on each participant. The Nidek optical AL Scan (Nidek co., Ltd, Japan) was used to measure the axial length. Only one normal eye per participant was included in the study.

The retinal nerve fibre layer and vertical disc height was measured using the Spectralis SD-OCT, Heidelberg eye explorer version 1.10.15.0, (Heidelberg Engineering, Germany). When performing the scan, the disc was identified with the aid of the built-in eye fixating system. The image was then tracked ensuring it remained within the image frame while maintaining a good quality scan. The scanning circle was manually placed over the optic nerve head and the images acquired. The participant values of each of the seven sectors (global, superotemporal, inferotemporal, superonasal, inferonasal, temporal, nasal) of the RNFL thickness were generated by the built-in software of the Heidelberg engineering. The participant values were displayed alongside the values of the European database which are automatically age- and gender-matched. Each sector is considered either "normal", "borderline", or "outside normal limits", if thicker than 5th centile, thinner than the 5th centile, or thinner than the 1st centile respectively, when compared to the European database of that sector [5]. Only good quality OCT scans $(\geq 21 \mathrm{~dB})$ and scans with the Fovea to Disc (FoDi) alignment within $7^{\circ}$, that were normal as defined by the age and gender matched European database, were included in the study. The reference value and participant results for each sector, as well as the quality of the scan and degree of cyclotorsion were documented. Patients whose FoDi alignment was outside $7^{\circ}$ on either side of the fovea, were excluded from the study. Vertical disc height (VDH) was measured manually by and agreed upon by two of the investigators (SI and NA). Data collected was captured on the REDCap database. Data was then exported to STATA 15.1 (STATACorp, USA) and analysed.

\section{Statistical analysis}

A sample size of hundred and twenty-eight patients was calculated using a one-sample mean test with a power of $80 \%$ and an $\alpha$-level of 0.05 to detect a difference of $2.5 \mu \mathrm{m}$ $(\mathrm{SD}=10)$ between the measured and reference values. The unpaired $t$-test was used to compare the mean RNFL thickness in the study population to the reference value per sector of the retina measured. A one sample proportion test was used to test the proportions of males and females within the study population. Univariate and multivariate 
Table 1 Comparison of RNFL thickness $(\mu \mathrm{m})$ in the study population vs reference database

\begin{tabular}{lllr}
\hline RNFL sector & $\begin{array}{l}\text { Mean RNFL thickness (SD) }(\mu \mathrm{m}) \\
{[95 \% \mathrm{CI}]}\end{array}$ & $\begin{array}{l}\text { Mean reference RNFL thickness }(\mathrm{SD}) \\
(\mu \mathrm{m})(95 \% \mathrm{CI})\end{array}$ & $p$ value $^{\mathrm{a}}$ \\
\hline Global & $108.7(10.7)[106.9-110.6]$ & $97.1(1.0)[96.9-97.2]$ & $<0.001$ \\
Superotemporal & $152.4(20.3)[148.9-155.9]$ & $134.6(2.1)[134.3-135.0]$ & $<0.001$ \\
Superonasal & $132.6(23.9)[128.5-136.7]$ & 102 & $<0.001$ \\
Inferotemporal & $150.1(19.7)[146.7-153.5]$ & $142.5(2.8)[141.9-143.0]$ & $<0.001$ \\
Inferonasal & $129.2(27.9)[124.4-134.0]$ & $105.9(1.1)[105.7-106.0]$ & $<0.001$ \\
Nasal & $77.7(14.6)[75.2-80.2]$ & 72 & $<0.001$ \\
Temporal & $74.8(10.3)[73.0-76.5]$ & $74.7(1.9)[73.9-75.6]$ & 0.9534 \\
\hline
\end{tabular}

${ }^{\mathrm{a}}$ Unpaired $t$-test (Bonferroni-corrected $p$ value $=0.0024$ ) linear regression models were used to assess the change in RNFL thickness with increasing age. The univariate and multivariate equations are shown below:

Univariate equation: $\mathrm{E}(\mathrm{RNFL})=\beta 0+\beta 1 \cdot$ age

Multivariate equation: $\mathrm{E}(\mathrm{RNFL})=\beta 0+\beta 1 \cdot$ age $+\beta 2 \cdot \mathrm{sex}$ $+\beta 3 \cdot \mathrm{vdh}$

Suitability for using a linear regression model was checked using the residual versus fitted plots. Since a multitude of statistical tests were performed on the same dataset, a Bonferroni correction was applied, and statistical significance was set at a $p$ value of 0.0024 .

\section{Results}

One hundred and thirty-two patients were enroled in this study. The mean(SD) age of patients in this study was 41.3 (12.5). The age range of patients in our study was 19-74 years. Males comprised $40.9 \%(n=54 ; p=0.0367)$ of patients in this study. The mean RNFL thickness in all sectors was significantly thicker when compared with the European reference RNFL thickness (Table 1). (Where no reference range is given in a reference RNFL sector, the Spectralis OCT machine gave a single value irrespective of gender and age). The mean (SD) [95\% CI] axial length and VDH were 23.5 (0.76) $\mathrm{mm}$ [23.3-23.6] and 1881.8 (185.2) $\mu \mathrm{m}$ [1849.9-1913.6] respectively.

In our study population the 1 st centile was thicker than the Heidelberg database 5th centile (Table 2). The univariate and multivariate regression co-efficients showed the decrease in RNFL thickness per decade increase in age (Table 3, Figs. 1, 2).

\section{Discussion}

Our study shows that the RNFL in the black South African population is significantly thicker than that of the European normative database on the Spectralis SD-OCT (Heidelberg
Table 2 Comparison between the 1 st and 5 th percentile of the Heidelberg database reference values [5] (45 years) and our study patients

\begin{tabular}{lllll}
\hline Sector & $\begin{array}{l}1 \text { st percentile }^{\mathrm{a}} \\
{[\mu \mathrm{m}]}\end{array}$ & $\begin{array}{l}1 \text { st percentile }^{\mathrm{b}} \\
{[\mu \mathrm{m}]}\end{array}$ & $\begin{array}{l}5 \text { th percentile }^{\mathrm{a}} \\
{[\mu \mathrm{m}]}\end{array}$ & $\begin{array}{l}5 \text { th percentile }^{\mathrm{b}} \\
{[\mu \mathrm{m}]}\end{array}$ \\
\hline Global & 87 & 76.0 & 93 & 82.1 \\
Temporal & 57 & 46.9 & 60 & 54.9 \\
Superotemporal & 111 & 96.3 & 119 & 107.4 \\
Inferotemporal & 117 & 99.1 & 122 & 111.6 \\
Nasal & 50 & 38.3 & 56 & 48.1 \\
Superonasal & 81 & 57.8 & 97 & 70.7 \\
Inferonasal & 84 & 53.6 & 90 & 68.8 \\
\hline
\end{tabular}

${ }^{\text {a }}$ Study values

${ }^{\mathrm{b}}$ Heidelberg database values

Engineering). The significance was maintained after applying a Bonferroni correction to the data. Although used primarily for glaucoma diagnosis, the RNFL thickness also has implications for the diagnosis of other neurological diseases such as multiple sclerosis and Alzheimer's disease [21]. In our study, the significant mean thickness difference, ranges from $5 \mu \mathrm{m}$ in the nasal sector to $30 \mu \mathrm{m}$ in the superonasal sector, with the higher differences being more clinically significant when used for glaucoma diagnosis. The Spectralis SD-OCT displays either a normal RNFL thickness; or "borderline" if it is thinner than the 5th percentile; or "outside normal limits" if it is thinner than the 1st percentile [5]. The Spectralis SDOCT gives normative values at age 45 and 60 years of age [5]. Using the Spectralis normative database values at age 45 years (mean age of our study population is 41.3 years), this difference results in the 1st percentile for our study eyes being thicker than the 5th percentile in the database (Table 3). This means that a patient who has structural damage that should be flagged as "outside normal limits" by the machine, will display as normal using the current normative database. We are potentially underdiagnosing a large proportion of patients with glaucoma.

The global RNFL thickness in our study population $(108.7 \mu \mathrm{m})$ is thicker than those of other populations measured using the Spectralis SD-OCT [6, 8-10, 16]. The global reference on the Spectralis SD-OCT, which is based on the 
Table 3 Change in RNFL thickness $(\mu \mathrm{m})$ per decade increase in age

\begin{tabular}{llrll}
\hline RNFL sector & $\begin{array}{l}\text { Unadjusted }{ }^{\mathrm{a}} \text { RNFL change per } \\
\text { decade }(95 \% \text { CI })\end{array}$ & $p$ value & $\begin{array}{l}\text { Adjusted }{ }^{\mathrm{b}} \text { RNFL change per } \\
\text { decade }(95 \% \mathrm{CI})\end{array}$ & $p$ value \\
\hline Global & $-3.5(-4.9$ to -2.2$)$ & $<0.001$ & $-3.5(-4.8$ to -2.1$)$ & $<0.001$ \\
Superotemporal & $-5.2(-7.9$ to -2.5$)$ & $<0.001$ & $-4.9(-7.6$ to -2.1$)$ & 0.001 \\
Superonasal & $-5.8(-8.9$ to -2.6$)$ & $<0.001$ & $-5.8(-9.0$ to -2.6$)$ & $<0.001$ \\
Inferotemporal & $-4.7(-7.3$ to -2.1$)$ & $<0.001$ & $-4.5(-7.1$ to -1.8$)$ & 0.001 \\
Inferonasal & $-3.4(-7.2$ to -0.3$)$ & 0.079 & $-3.3(-7.1$ to 0.4$)$ & 0.080 \\
Nasal & $-2.0(-4.0$ to -0.02$)$ & $0.047-2.4(-4.4$ to -0.3$)$ & 0.046 \\
Temporal & $-2.5(-3.8$ to -1.1$)$ & $<0.001$ & $-2.3(-3.7$ to -0.9$)$ & 0.002 \\
\hline
\end{tabular}

${ }^{\mathrm{a}}$ Univariate linear regression (Bonferroni-corrected $p$ value $=0.0024$ ) ${ }^{\mathrm{b}}$ Multivariate linear regression (Bonferroni-corrected $p$ value $=0.0024$ )

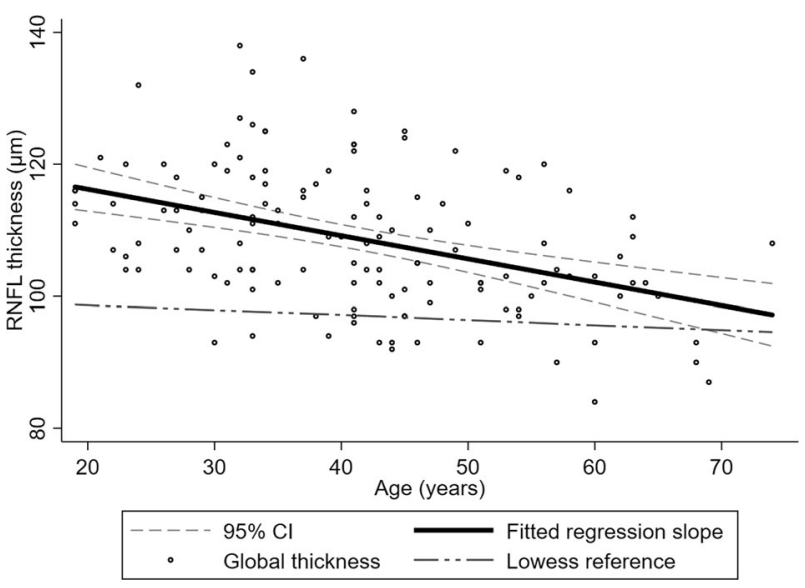

Fig. 1 Composite graph of fitted regression slope with 95\% confidence interval for global RNFL thickness vs age (thick black line straddled by two dashed lines), scatterplot of measured global RNFL thickness datapoints (hollow circles), and LOWESS curve of the reference database values (dash-dots line)

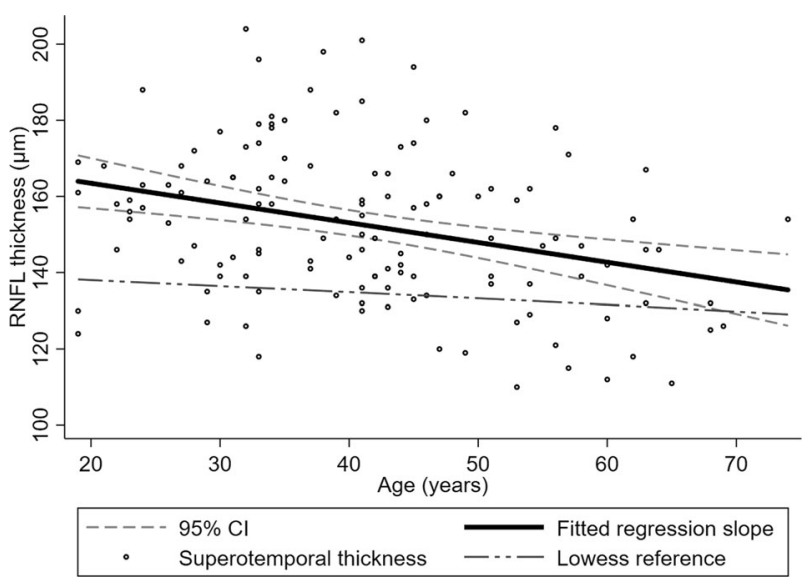

Fig. 2 Composite graph of fitted regression slope with $95 \%$ confidence interval for superotemporal RNFL thickness vs age (thick black line straddled by two dashed lines), scatterplot of measured superotemporal RNFL thickness datapoints (hollow circles), and LOWESS curve of the reference database values (dash-dots line)

European database is $97.1 \mu \mathrm{m}$. In Indian, Nepalese and Brazilian populations, the global RNFL thickness measured
101.43, 102.64 and 102/103 $\mu \mathrm{m}$ respectively [8-10]. This racial difference between the European and non-European RNFL thickness is therefore not unique to African patients. Bendschneider et al. demonstrated a global RNFL thickness in a German population of $97.2 \mu \mathrm{m}$ which is similar to the Heidelberg normative global mean thickness [6]. One of the few studies that used the Spectralis SD-OCT to measure the RNFL in a black population was conducted by Alasil et al. [7]. They measured RNFL thickness of various ethnicities in an American population [7]. Of those enroled, only 26 patients were of African-American descent and the global RNFL measured with the Spectralis SD-OCT was $99 \mu \mathrm{m}$ which is thinner than our population findings. The study consisted of 125 Caucasian patients and no significant difference was found between the RNFL thickness of the African-American and Caucasian population groups [7]. The French Alienor study measured RNFL thickness in elderly patients with a mean age of 81 and showed a global RNFL thickness of $91 \mu \mathrm{m}$ [16]. The mean age difference between the French Alienor study population (81 years) and ours (41.3 years), probably accounts for the large differences between mean RNFL thickness.

RNFL thickness can be influenced by factors other than the amount of nerve fibre layer tissue present in the peripapillary area, such as, vascular tissue as documented by OCTA [22]. The difference between the African RNFL thickness and European thickness could therefore be due to anatomical differences in blood vessel volume between these patients. Similarly, patients with larger disc areas have been shown to have a thicker RNFL [23]. Even though our study did not look at disc area, we have adjusted for vertical disc height in our multivariate analysis and we found it to be significant in predicting RNFL decline per decade. Black patients have also been shown to have larger disc areas when compared to their European counterparts [24]. This difference may also account for the thicker RNFL in our study.

The mean global RNFL thickness in two different Nigerian population groups conducted with the Stratus OCT were 104.1 and $107.1 \mu \mathrm{m}$ respectively $[17,18]$. Other South African studies also yielded mean values of $108-110 \mu \mathrm{m}$ 
$[11,19]$. However, even though the absolute values are similar to those in our study, different OCT machines were used and thus are not directly comparable [15].

Our study consisted of a wide range of patient ages and we were able to model univariate and multivariate linear regression models. After adjusting for age, gender and VDH in the multivariate linear regression analysis, the rate of decline in global RNFL thickness in our population is $-3.9 \mu \mathrm{m}$ per decade which is higher than that of other comparable studies. We also found that although age and VDH was a significant predictor in the multivariate model, gender was not a significant predictor. The rate of decline per decade in the Indian population was $-1.5 \mu \mathrm{m}$ and the Nepalese population was $-2.26 \mu \mathrm{m}[9,10]$. The rate of decline in the German population was $-1.9 \mu \mathrm{m}$ per decade [6]. Only the French Alienor study had a higher rate of decline of $-5.9 \mu \mathrm{m}$ but this is probably due to the older age of the participants. The higher rate of RNFL loss among our study patients' needs to be investigated further using longitudinal studies. Since glaucoma causes accelerated thinning of the RNFL, this high rate of loss in normal patients is probably even higher in glaucoma patients and may be the reason for increased severity of disease among African patients.

Bowd et al. recently published a prospective longitudinal study, which included participants enroled in the ADAGES and DIGS trials, comparing the RNFL thickness and BMOMRW in African and European patients in 3 categories (Healthy eyes, glaucoma suspect, and glaucomatous eyes) [25]. Only 27 of the 124 healthy eyes studied were from patients of African descent who had a mean age of 64 years. The global RNFL thickness measured by Spectralis SDOCT in these patients was $96.1 \mu \mathrm{m}$ and is much lower than our African population studied. This is probably due to the older age group of the participants. The rate of decline over 3 years was measured to be $-5.1 \mu \mathrm{m}$ [25]. This is a higher rate of decline compared with our population.

A thicker RNFL has been associated with a faster rate of decline in RNFL thickness [26]. After adjusting for age and sex in the multivariate regression model, the thicker quadrants (superonasal, inferotemporal, superotemporal) were noted to have the largest rates of decline in RNFL thickness. Thus, the larger rate of decline per decade could be attributed to the thicker baseline RNFL in our population group.

The limitations of the study are that this was a hospitalbased, cross-sectional study as opposed to a populationbased longitudinal cohort study. There may be inherent bias in recruiting hospital patients for a normative database. However, the difference in the superior and inferior quadrants compared to the normative database are both statistically and clinically significant. BMO-MRW was not measured in this study and could be done in future studies in African patients. BMO-MRW requires more cooperation from the patient and takes longer to obtain than RNFL. Reznicek et al. showed that RNFL and BMO-MRW are both suitable for the diagnosis of glaucoma, however, BMO-MRW may be more suitable in myopic patients [27] Further longitudinal studies of African patients need to be conducted to corroborate these results.

\section{Conclusion}

RNFL thickness in a black South African population, measured by Spectralis SD-OCT is both clinically and statistically significantly thicker than the European database. The difference in the 1st and 5th percentiles of our study and the built-in database are large enough when compared, to warrant requiring African-specific databases to avoid underdiagnosis of glaucoma and other diseases causing thinning of the RNFL. The rate of decline in RNFL thickness per decade increase in age, is greater than other comparable studies. Further, multicentre, multiregional, longitudinal population-based studies are however required to confirm these findings in black patients.

\section{Summary}

\section{What was known before}

- No studies of black African population using Spectralis SD-OCT.

- African-American subjects were shown to have no difference in RNFL thickness.

- Studies on other race groups e.g. Nepalese showed a thicker RNFL compared to Caucasian counterparts.

\section{What this study adds}

- The black South African population have a significantly thicker RNFL.

- This can result in glaucoma being missed.

- There is also a higher rate of decline in RNFL thickness of the black South African population.

\section{Compliance with ethical standards}

Conflict of interest The authors declare that they have no conflict of interest.

Publisher's note Springer Nature remains neutral with regard to jurisdictional claims in published maps and institutional affiliations. 


\section{References}

1. Tham Y-C, et al. Global prevalence of glaucoma and projections of glaucoma burden through 2040. Ophthalmology. 2014;121: 2081-90.

2. Sample PA, et al. The African Descent and Glaucoma Evaluation Study (ADAGES): design and baseline data. Arch Ophthalmol. 2009;127:1136-45.

3. Tielsch JM, et al. Racial variations in the prevalence of primary open-angle glaucoma: the Baltimore Eye Survey. JAMA. 1991;266:369-74.

4. Tao Y-L, et al. Parameters of ocular fundus on spectral-domain optical coherence tomography for glaucoma diagnosis. Int $\mathbf{J}$ Ophthalmol. 2017;10:982-91.

5. Downloads-SPECTRALIS OCT Angiography Module - Noninvasive imaging I Heidelberg Engineering. https://businesslounge.heidelbergengineering.com/za/en/products/spectralis/oct-a ngiography-module/downloads/\#downloads. Accessed 30 Mar 2018.

6. Bendschneider $\mathrm{D}$, et al. Retinal nerve fiber layer thickness in normals measured by spectral domain OCT. J Glaucoma. 2010; 19:475-82.

7. Alasil $\mathrm{T}$, et al. Analysis of normal retinal nerve fiber layer thickness by age, sex, and race using spectral domain optical coherence tomography. J Glaucoma. 2013;22:532-41.

8. Zangalli CES, Reis ASC, Vianna JR, Vasconcellos JPC, Costa VP. Interocular asymmetry of minimum rim width and retinal nerve fiber layer thickness in healthy Brazilian individuals. J Glaucoma. 2018;27:1136-41.

9. Appukuttan B, Giridhar A, Gopalakrishnan M, Sivaprasad S. Normative spectral domain optical coherence tomography data on macular and retinal nerve fiber layer thickness in Indians. Indian $\mathbf{J}$ Ophthalmol 2014;62:316-21.

10. Thapa M, Khanal S, Shrestha GB, Sharma AK. Retinal nerve fibre layer thickness in a healthy Nepalese population by spectral domain optical coherence tomography. Nepal J Ophthalmol. 2014;6:131-9.

11. Mashige KP, Oduntan OA. Retinal nerve fibre layer thickness values and their associations with ocular and systemic parameters in Black South Africans. Afr Health Sci. 2016;16:1188-94.

12. Savini G, Carbonelli M, Barboni P. Retinal nerve fiber layer thickness measurement by Fourier-domain optical coherence tomography: a comparison between Cirrus-HD OCT and RTVue in healthy eyes. J Glaucoma. 2010;19:369-72.

13. Seibold LK, Mandava N, Kahook MY. Comparison of retinal nerve fiber layer thickness in normal eyes using time-domain and spectral-domain optical coherence tomography. Am J Ophthalmol 2010;150:807-14.
14. Knight OJ, Chang RT, Feuer WJ, Budenz DL. Comparison of retinal nerve fiber layer measurements using time domain and spectral domain optical coherent tomography. Ophthalmology. 2009;116:1271-7.

15. Arthur SN, et al. Reproducibility and agreement in evaluating retinal nerve fibre layer thickness between Stratus and Spectralis OCT. Eye. 2011;25:192-200.

16. Rougier M-B, et al. Retinal nerve fibre layer thickness measured with SD-OCT in a population-based study of French elderly subjects: the Alienor study. Acta Ophthalmol. 2015;93:539-45.

17. Sani RY, Abdu L, Pam V. Retinal nerve fiber layer thickness measurements of normal Northern Nigerian adults using optical coherence tomography. Ann Afr Med. 2016;15:52-57.

18. Mahmud-Ajeigbe. Retinal nerve fibre layer measurements in normal eyes in Zaria using optical coherence tomography. http://www.ssajm.org/article.asp?issn =2384-5147; year=2015; volume $=2$; issue $=2$; page $=85$; epage $=88$; aulast $=$ MahmudAjeigbe. Accessed 29 Jan 2019.

19. Murugan, $C$ et al. Retinal nerve fibre layer thickness of black and Indian myopic students at the University of KwaZulu-Natal. Afr. Vis. Eye Health. 2015;74:1-6.

20. Pathai, $S$ et al. Retinal nerve fibre layer thickness and contrast sensitivity in HIV-infected individuals in South Africa: a casecontrol study. PLoS ONE. 2013;8:e73694.

21. Gupta S, Zivadinov R, Ramanathan M, Weinstock-Guttman B. Optical coherence tomography and neurodegeneration: are eyes the windows to the brain? Exp Rev Neurother 2016;16: 765-75.

22. Allegrini D, et al. The volume of peripapillary vessels within the retinal nerve fibre layer: an optical coherence tomography angiography study of normal subjects. Br J Ophthalmol 2018;102:611-21.

23. Gür Güngör S, Akman A, Küçüködük A, Çolak M. Retinal nerve fiber layer thicknesses in three different optic nerve head size groups measured by cirrus spectral domain optical coherence tomography. Turk J Ophthalmol. 2016;46:58-61.

24. Oliveira $\mathrm{C}$, et al. Axial length and optic disc size in normal eyes. Br J Ophthalmol 2007;91:37-39.

25. Bowd C, et al. Racial differences in rate of change of spectraldomain optical coherence tomography-measured minimum rim width and retinal nerve fiber layer thickness. Am J Ophthalmol 2018;196:154-64.

26. Leung CKS, et al. Retinal nerve fiber layer imaging with spectraldomain optical coherence tomography: a prospective analysis of age-related loss. Ophthalmology. 2012;119:731-7.

27. Reznicek L, et al. Structure-function relationship comparison between retinal nerve fibre layer and Bruch's membrane openingminimum rim width in glaucoma. Int J Ophthalmol. 2017;10: $1534-8$. 